\title{
Editorial: The Evolution of the Stratospheric Ozone
}

\author{
Gabriel Chiodo ${ }^{1,2}$, Jane Liu $^{3}$, Laura Revell ${ }^{4}$, Timofei Sukhodolov ${ }^{5,6,7 *}$ and Jiankai Zhang ${ }^{8}$ \\ ${ }^{1}$ Institute for Atmospheric and Climate Science, ETH Zurich, Zurich, Switzerland, ${ }^{2}$ Department of Applied Physics and Applied \\ Mathematics, Columbia University, New York, NY, United States, ${ }^{3}$ Department of Geography and Planning, University of Toronto, \\ Toronto, ON, Canada, ${ }^{4}$ School of Physical and Chemical Sciences, University of Canterbury, Christchurch, New Zealand, \\ ${ }^{5}$ Physikalisch-Meteorologisches Observatorium Davos and World Radiation Center, Davos, Switzerland, ${ }^{6}$ St. Petersburg State \\ University, St. Petersburg, Russia, ${ }^{7}$ Institute of Meteorology and Climatology, University of Natural Resources and Life Sciences, \\ Vienna, Austria, ${ }^{8}$ College of Atmospheric Sciences, Lanzhou University, Lanzhou, China
}

Keywords: ozone layer, middle atmosphere, atmospheric chemistry, stratosphere, chemistry-climate modelling

\section{Editorial on the Research Topic}

\section{The Evolution of the Stratospheric Ozone}

The evolution of the stratospheric ozone layer remains a central problem to contemporary science because of its importance for the sustainable development of human society. Besides its role in shielding the biosphere from dangerous solar UV radiation, it is now recognized that stratospheric ozone plays an important role in the global atmosphere and climate variability. The variability of the ozone layer is related to many atmospheric processes including chemistry, radiation, and dynamics, which are all coupled on different time scales. In the 1980s, it was discovered that the ozone layer was being damaged by anthropogenic halocarbons, which led to limitations on the production of halocarbon-containing ozone depleting substances via the Montreal Protocol and its Amendments. Numerous studies have confirmed the substantial positive role of the Montreal Protocol for climate and ozone layer and the rebound of the total column ozone (TCO) is now clear and can be seen even at individual locations, such as the Arosa station in Switzerland (Figure 1).

This Special Topic includes papers focused on a range of topics relating to the ozone layer. Several papers addressed trends in ozone in models and observations, while others were more processoriented and focused on specific chemical and dynamical mechanisms. Some papers also covered potential links of ozone science advances to adjacent fields like using Arctic ozone as a source of information for seasonal forecasting or investigating computationally inexpensive ways to project or reconstruct the ozone layer.

Rozanov et al. analyzed the world's longest TCO measurement series in Arosa/Davos (Switzerland) in terms of its representativeness for different spatial and temporal scales. Using the satellite, historical reanalysis, and modelling data, they showed that this time series correlates very well with the global and northern hemispheric means and can serve as a useful proxy for statistical reconstructions of global ozone behavior and monitoring long-term trends, as well as model evaluation. Zou et al. looked specifically at the trends over the Tibetan Plateau, a region that was found to have a lower value of TCO compared to the other regions in the same latitudes, which is called the Tibetan ozone deficit. Using ozone satellite data, they confirmed that there is an overall positive trend in TCO in these latitudes since 2005, and that the ozone layer over the Tibetan Plateau is healing faster than its zonal average since 2009, thus making this region less vulnerable to the UV radiation. The physical mechanism for these changes is still to be investigated. Future model projections were analyzed in Keeble et al., who focused on simulations under different Shared Socioeconomic Pathways (SSPs) using the UKESM1 Earth system model. Global stratospheric column ozone was projected to be largest in 2,100 under SSP scenarios with the smallest change in 


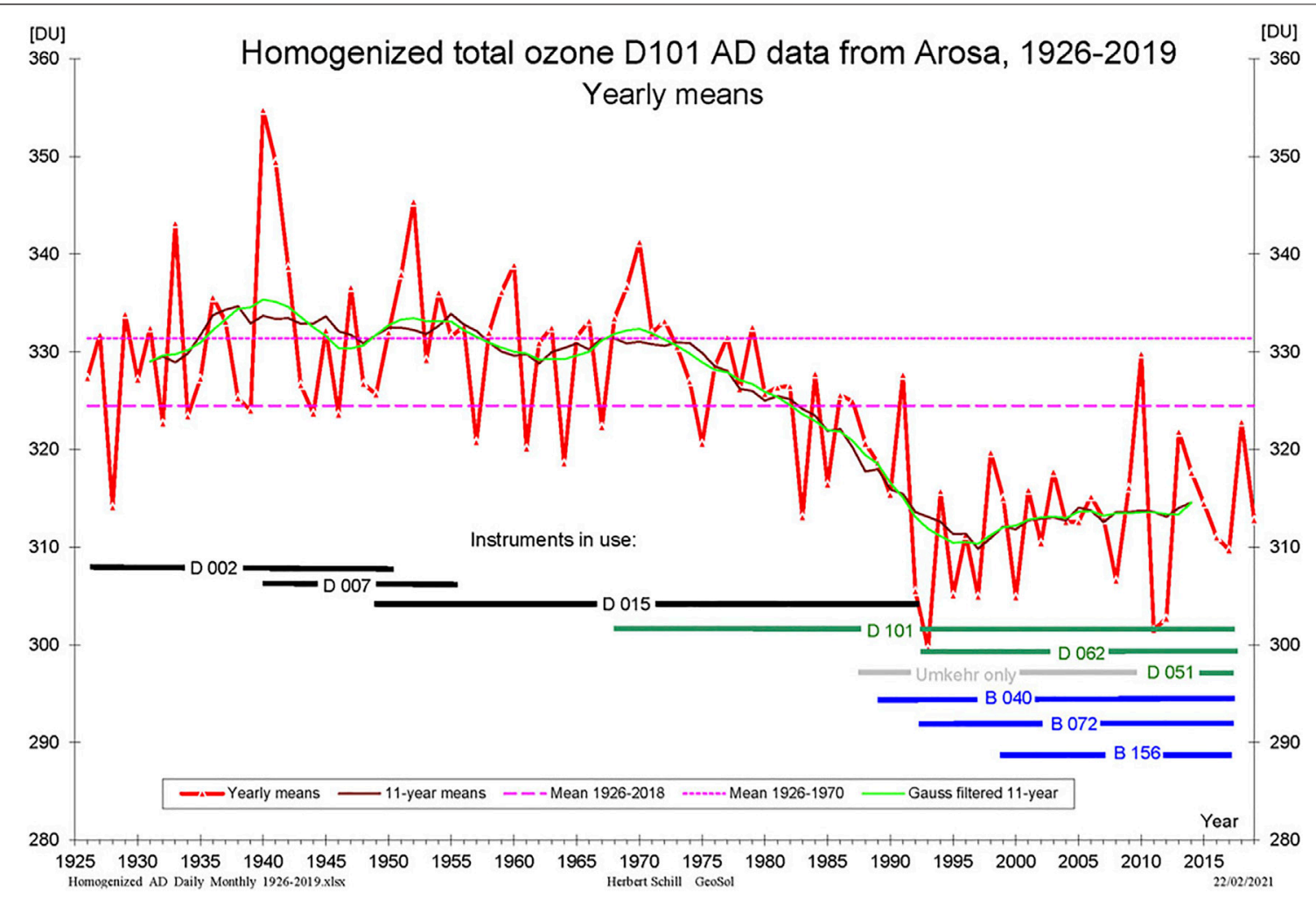

FIGURE 1 | Homogenized total column ozone from Arosa and Davos (Switzerland), 1926-2019. Adapted from Rozanov et al.

radiative forcing, and smallest for SSP scenarios with larger radiative forcing. They also investigated the application of computationally inexpensive machine learning techniques for future projections of tropical ozone, and showed that, in general, they do a good job for low and mid-range SSP scenarios. Therefore further development of these tools has the potential to supplement future scenarios by more complex chemistry climate models, thus reducing the computational burden posed by them.

Mironova et al. studied the effect of decreased forcing by galactic cosmic rays during the geomagnetic storms of January 2005, known as the Forbush effect. Based on simulations with the chemistry-climate model SOCOLv2, they found that concentrations of the hydrogen oxide radical were reduced by $50 \%$ over the polar boreal stratosphere owing to a decrease in ionization rates during the events. Robust responses in NOx and ozone were not found. Nevertheless, this study provides important insights for the cases of stronger solar events. Another important contribution to the general knowledge of the processes affecting the ozone layer was provided by Klobas et al., who looked at the atmospheric iodine cycle, a component which is typically omitted in chemistry-climate models. Until recently, iodine was not thought to enter the stratosphere due to its short tropospheric lifetime, however recent research has shown that heterogeneous processes may enhance the efficiency of iodine injection into the stratosphere, and that atmospheric iodine concentrations may be increasing in response to human activities. Klobas et al. evaluated the importance of iodinecatalysed ozone loss under different greenhouse gas emissions scenarios and found that, in comparison to chlorine- and bromine-catalysed reactions, iodine-catalysed ozone loss is less sensitive to long-term changes in stratospheric dynamics and composition. Due to the expected decrease in halocarbons, the relative importance of iodine-catalysed ozone loss might also increase over coming decades.

Two papers looked closely at the Asian summer monsoon anticyclone (ASMA), which is a significant part of global stratosphere-troposphere exchange (STE). ASMA is of importance for the ozone layer, as it controls the crosstropopause transport of ozone and other species. Vertical velocity in the upper troposphere and lower stratosphere (UTLS), however, is highly uncertain, which affects the accurate estimation of STE over the ASMA region. Guo et al. performed calculations of the local vertical velocities using a thermodynamic method based on high-resolution heating rates calculated from the observed in situ profiles of ozone and water vapor. They suggested that their method may better capture the descending motion in the west and north and ascending in the east and south over the Tibetan Plateau in summer. Besides the impact of the vertical motions on STE, gravity waves may also influence the STE processes. Chang et al. addressed this issue through the analysis 
of a topographic gravity wave event in May 2014 by using the ERA5 reanalysis data and Weather Research and Forecasting (WRF) mesoscale model. They found that increased gravity waves breaking intensified the STE causing the ozone-rich air intrusion from the stratosphere to the upper troposphere. Overall, their work highlights the importance of small-scale processes in driving STE and more generally UTLS composition.

Besides stratospheric air intrusions, it has been recognized that stratospheric ozone can also impact the troposphere by modulating the vertical dynamical coupling and the high-latitude weather and climate in the troposphere. Xie and Ma presented an example of such influences. Their study, for the first time, found that Arctic stratospheric ozone (ASO) can be an effective and robust predictor for April precipitation in the northwestern United States with a 1month lead. The possible mechanisms, as they suggested, are that March ASO changes can cause changes in the jet-stream over the Pacific, which can have impacts further downstream, including precipitation in the Northeastern US.

We thank the authors and reviewers who contributed to this Special Topic. Together these papers provide valuable insight into the evolution of stratospheric ozone in the coming century, and open up exciting avenues for future research.

\section{AUTHOR CONTRIBUTIONS}

All authors listed have made a substantial, direct, and intellectual contribution to the work and approved it for publication.

\section{FUNDING}

TS work was supported by the Swiss National Science Foundation (SNSF) (POLE, grant no. 200020-182239) and was partly performed in the SPbSU "Ozone Layer and Upper Atmosphere Research" laboratory supported by the Ministry of Science and Higher Education of the Russian Federation under Grant 075-15-2021583. JZ was supported by the National Natural Science Foundation of China (42075062) and the Fundamental Research Funds for the Central Universities (lzujbky-2021-ey04). GC was supported by the SNSF Ambizione grant PZ00P2_180043. LR's work was supported by the Ministry of Business, Innovation and Employment programme "Extreme events and the emergence of climate change" (E3574).

Conflict of Interest: The authors declare that the research was conducted in the absence of any commercial or financial relationships that could be construed as a potential conflict of interest.

Publisher's Note: All claims expressed in this article are solely those of the authors and do not necessarily represent those of their affiliated organizations, or those of the publisher, the editors and the reviewers. Any product that may be evaluated in this article, or claim that may be made by its manufacturer, is not guaranteed or endorsed by the publisher.

Copyright $\odot 2021$ Chiodo, Liu, Revell, Sukhodolov and Zhang. This is an open-access article distributed under the terms of the Creative Commons Attribution License (CC $B Y)$. The use, distribution or reproduction in other forums is permitted, provided the original author(s) and the copyright owner(s) are credited and that the original publication in this journal is cited, in accordance with accepted academic practice. No use, distribution or reproduction is permitted which does not comply with these terms. 